\title{
MORAN SETS AND HYPERBOLIC BOUNDARIES
}

\author{
Jun Jason Luo \\ Shantou University, Department of Mathematics \\ Shantou 515063, P. R. China; luojun2011@yahoo.com.cn
}

\begin{abstract}
In the paper, we prove that a Moran set is homeomorphic to the hyperbolic boundary of the representing symbolic space in the sense of Gromov, which generalizes the results of Lau and Wang [10]. Moreover, by making use of this, we establish the Lipschitz equivalence of a class of Moran sets.
\end{abstract}

\section{Introduction}

For an iterated function system (IFS) of contractive similitudes on $\mathbf{R}^{d}$ and the associated self-similar set $K$, there is a symbolic space which contains a natural tree structure and represents every point of $K$. Kaimanovich [6] first proposed a hyperbolic graph structure (called augmented tree) on the symbolic space of the Sierpinski gasket by adding horizontal edges (corresponding to the (vertical) edges of the tree), and he observed the relationship between the hyperbolic boundary and the gasket. Lau and Wang $([10,14])$ developed this idea to more general self-similar sets. It was proved that if an IFS satisfies the open set condition (OSC), even weak separation condition (WSC), then the augmented tree is hyperbolic in the sense of Gromov and the hyperbolic boundary of the tree is shown to be homeomorphic to $K$; moreover under certain mild condition, the homeomorphism is actually a Hölder equivalent map. Recently, this setup has been frequently used to study the random walks on trees and their Martin boundaries $([7,5,11])$ and Lipschitz equivalence problem [8].

As generalizations of self-similar sets, Moran sets were introduced by Moran [13], which have abundant exotic fractal structures. Let $\left\{n_{k}\right\}_{k \geq 1}$ be a sequence of positive integers and $\left\{r_{k}\right\}_{k \geq 1}$ be a sequence of positive numbers satisfying $n_{k} \geq 2,0<r_{k}<1$ and $n_{k} r_{k} \leq 1$. For any $k \geq 1$, let $D_{k}=\prod_{j=1}^{k}\left\{1,2, \ldots, n_{j}\right\}=\left\{i_{1} \cdots i_{k}: 1 \leq i_{j} \leq\right.$ $\left.n_{j}, 1 \leq j \leq k\right\}$ be the set of words (or $k$-multi-indexes) and $D=\bigcup_{k>0} D_{k}$ be the set of all finite words (convention $D_{0}=\emptyset$ ), and let $D_{\infty}=\left\{i_{1} i_{2} \cdots: 1 \leq i_{j} \leq n_{j}, j=\right.$ $1,2, \ldots\}$ be the set of all infinite words. For integers $\ell>k \geq 1$, if $\mathbf{i}=i_{1} \cdots i_{k} \in D_{k}$ and $\mathbf{j}=j_{1} \cdots j_{m} \in \prod_{j=k+1}^{\ell}\left\{1,2, \ldots, n_{j}\right\}$, we denote by $\mathbf{i j}=i_{1} \cdots i_{k} j_{1} \cdots j_{m} \in D_{\ell}$ the concatenation.

Definition 1.1. Suppose that $J \subset \mathbf{R}^{d}$ is a compact set with nonempty interior. The collection of subsets $\mathcal{F}=\left\{J_{\mathbf{i}}: \mathbf{i} \in D\right\}$ of $J$ has the Moran structure, if it satisfies:

(i) $J_{\emptyset}=J$;

(ii) for any $\mathbf{i} \in D, J_{\mathbf{i}}$ is geometrically similar to $J$, that is, there exists a similarity $S_{\mathbf{i}}: \mathbf{R}^{d} \rightarrow \mathbf{R}^{d}$ such that $J_{\mathbf{i}}=S_{\mathbf{i}}(J) ;$

doi:10.5186/aasfm.2013.3814

2010 Mathematics Subject Classification: Primary 28A80.

Key words: Moran set, augmented tree, hyperbolic boundary, Lipschitz equivalence.

The research is supported by STU Scientific Research Foundation for Talents (no. NTF12016). 
(iii) for any $k \geq 1$ and $\mathbf{i} \in D_{k-1}, J_{\mathbf{i} 1}, \ldots, J_{\mathbf{i} n_{k}}$ are subsets of $J_{\mathbf{i}}$ and $\operatorname{int}\left(J_{\mathbf{i} i}\right) \cap$ $\operatorname{int}\left(J_{\mathbf{i} j}\right)=\emptyset$ for $i \neq j$ where $\operatorname{int}(A)$ denotes the interior of a set $A$;

(iv) for any $k \geq 1$ and $\mathbf{i} \in D_{k-1}, 1 \leq j \leq n_{k}$, we have

$$
\frac{\left|J_{\mathbf{i} j}\right|}{\left|J_{\mathbf{i}}\right|}=r_{k}
$$

where $|A|$ denotes the diameter of $A$.

We call $E:=\bigcap_{k \geq 0} \bigcup_{\mathbf{i} \in D_{k}} J_{\mathbf{i}}$ the (homogeneous) Moran set. For $\mathbf{i} \in D_{k-1}$ with $k \geq 1$, we call $J_{\mathbf{i}}$ a basic set of order $k$ of the Moran set. Let $\mathcal{M}:=\mathcal{M}\left(J,\left\{n_{k}\right\},\left\{r_{k}\right\}\right)$ denote the class of the Moran sets satisfying (i)-(iv). From the definition above, if the positions of the basic sets are different, then the Moran sets are different. Compared with self-similar sets, Moran sets have more fractal structures as following:

(1) the placements of the basic sets at each step of the geometric construction can be arbitrary;

(2) the contraction ratios can be different at different steps;

(3) the cardinality of the basic sets in replacement at different steps can be different.

The systematical study on the geometric structure and dimension theory of Moran sets was developed by $[2,4,15]$. It is well-known that all Moran sets in $\mathcal{M}$ have the same Hausdorff and packing dimensions provided $r:=\inf _{k} r_{k}>0$ [2]. In our consideration we always assume this condition holds.

However, the relationship between Moran sets and the hyperbolic structures of the representing symbolic spaces has not been established yet. Similarly to the selfsimilar set, we can define the corresponding symbolic space and augmented tree of a Moran set or class. Let

$$
X_{n}=\left\{i_{1} \cdots i_{k} \in D: r_{1} \cdots r_{k} \leq r^{n}<r_{1} \cdots r_{k-1}\right\} .
$$

Denote $X=\bigcup_{n>0} X_{n}$, where $X_{0}=\emptyset$. Note that for $n \geq 0, n \neq \ell, X_{n} \cap X_{\ell}=\emptyset$ holds. For each word $\mathbf{i} \in X_{n}$, there exists a unique word $\mathbf{j} \in X_{n-1}$ and a multi-index $\mathbf{k}=k_{1} \cdots k_{\ell}$ such that $\mathbf{i}=\mathbf{j k}$. Then we can define a vertical edge in this way, the set of all vertical edges is denoted by $\mathcal{E}_{v}$. The horizontal edge is defined as follows: $\mathbf{i} \sim \mathbf{j}$ is called a horizontal edge if for some $n \geq 1$ and $\mathbf{i}, \mathbf{j} \in X_{n}, J_{\mathbf{i}} \cap J_{\mathbf{j}} \neq \emptyset$, the set of all horizontal edges is denoted by $\mathcal{E}_{h}$. Let $\mathcal{E}=\mathcal{E}_{v} \cup \mathcal{E}_{h}$, then we call $(X, \mathcal{E})$ an augmented tree (in Kaimanovich's sense [6]) induced by the triplet $\left(J,\left\{n_{k}\right\},\left\{r_{k}\right\}\right.$ ) (or a Moran set $E \in \mathcal{M}$ ). There is a hyperbolic metric $\rho_{a}$ (see Section 2) on $X$ which induces a hyperbolic boundary $\partial X:=\hat{X} \backslash X$ where $\hat{X}$ is the completion of $X$ under the $\rho_{a}$.

One of the main purposes of the present paper is to extend Lau and Wang's results on self-similar sets [10] to that on Moran sets as following.

Theorem 1.2. Let $E$ be a Moran set, $(X, \mathcal{E})$ be the induced augmented tree. Then

(i) $(X, \mathcal{E})$ is a hyperbolic graph in the sense of Gromov.

(ii) $E$ is homeomorphic to the hyperbolic boundary $\partial(X, \mathcal{E})$. Furthermore, the Hölder equivalence holds if we assume the additional condition $(H)$ (see Section 3).

In [8], we investigated the Lipschitz equivalence of self-similar sets and self-affine sets by employing the structure of the augmented tree and its hyperbolic boundary. 
Based on the same goal, in this paper, we also establish the Lipschitz equivalence relationship for a class of Moran sets.

Let $J \subset \mathbf{R}^{d}$ be a connected compact set with $\operatorname{int}(J) \neq \emptyset$ and let $r_{k} \equiv r \in(0,1)$ in Definition 1.1. In this case, $X_{n}=D_{n}$ for $n \geq 0$. We define a special Moran class $\mathcal{M}^{\prime}:=\mathcal{M}\left(J,\left\{n_{k}\right\}, r\right)$ if in addition two more conditions hold:

(v) There exists $L \in \mathbf{N}$ such that for any $k \geq 1$ and a subset $T \subset D_{k-1}$, if $\bigcup_{\mathbf{i} \in T} J_{\mathbf{i}}$ is a connected component of $\bigcup_{\mathbf{i} \in D_{k-1}} J_{\mathbf{i}}$ topologically, then $\# T \leq L$.

(vi) For any $k \geq 1, T \subset D_{k-1}$ and $\mathbf{i} \in T$, if $J_{\mathbf{i} 1}, \ldots, J_{\mathbf{i} n_{k}}$ are subsets of $J_{\mathbf{i}}$ in the next step, then the union of all subsets of $\bigcup_{\mathbf{i} \in T} J_{\mathbf{i}}$ can be written as $\# T(:=b)$ disjoint groups as follows

$$
\bigcup_{\mathbf{i} \in T} \bigcup_{j=1}^{n_{k}} J_{\mathbf{i} j}=\left(\bigcup_{k \in \Lambda_{1}} C_{k}\right) \cup \cdots \cup\left(\bigcup_{k \in \Lambda_{b}} C_{k}\right)
$$

where $C_{k}$ are connected components of $\bigcup_{\mathbf{i} \in T} \bigcup_{j=1}^{n_{k}} J_{\mathbf{i} j}$ topologically such that every group contains exactly $n_{k}$ terms of $J_{\mathbf{i} j}$.

Since the Moran structure closely depends on the positions of the basic sets in the constructing process, we can choose proper basic sets in each step to satisfy conditions (v) and (vi). For example, the fractal sets generated by a sequence of nested intervals or squares (see $[12,17]$ ).

Theorem 1.3. For any two Moran sets $E, F \in \mathcal{M}^{\prime}$ defined as above. If the condition in (ii) of Theorem 1.2 holds, then $E$ and $F$ are Lipschitz equivalent.

Actually we prove a less restrictive form of Theorem 1.3 (Theorem 4.6) in terms of the rearrangeable augmented tree.

The rest of the paper is organized as follows. In Section 2, we recall some wellknown results about hyperbolic graphs. In Section 3, we identify Moran sets with hyperbolic boundaries and prove Theorem 1.2. We introduce a concept of 'rearrangeable augmented tree' to prove Theorems 1.3 in Section 4 .

\section{Hyperbolic graphs}

Let $X$ be a countably infinite set, we say that $X$ is a graph if it is associated with a symmetric subset $\mathcal{E}$ of $(X \times X) \backslash\{(x, x): x \in X\}$, and call $x \in X$ a vertex, $(x, y) \in \mathcal{E}$ an edge, which is more conveniently denoted by $x \sim y$ (intuitively, $x, y$ are neighborhood to each other). By a path in $X$ from $x$ to $y$, we mean a finite sequence $x=x_{0}, x_{1}, \ldots, x_{n}=y$ such that $x \sim x_{i+1}, i=0, \ldots, n-1$. We always assume that the graph $X$ is connected, i.e., there is a path joining any two vertices $x, y \in X$. We call $X$ a tree if the path between any two points is unique. We equip a graph $X$ with an integer-valued metric $d(x, y)$, which is the minimum among the lengths of the paths from $x$ to $y$, and denote the corresponding geodesic path by $\pi(x, y)$. We also use $|\pi(x, y)|$ to be the length of the geodesic, which equals $d(x, y)$. Let $o \in X$ be a fixed point in $X$ and call it the root of the graph. We use $|x|$ to denote $d(o, x)$, and say that $x$ belongs to $n$-th level if $d(o, x)=n$.

A graph $X$ is called hyperbolic (with respect to $o$ ) if there is $\delta>0$ such that

$$
|x \wedge y| \geq \min \{|x \wedge z|,|z \wedge y|\}-\delta \quad \forall x, y, z \in X
$$


where $|x \wedge y|:=\frac{1}{2}(|x|+|y|-d(x, y))$ is the Gromov product $[3,16]$. For a fixed $a>0$ with $a^{\prime}=\exp (\delta a)-1<\sqrt{2}-1$, we define a hyperbolic metric $\rho_{a}(\cdot, \cdot)$ on $X$ by

$$
\rho_{a}(x, y)= \begin{cases}\exp (-a|x \wedge y|) & \text { if } x \neq y \\ 0 & \text { otherwise }\end{cases}
$$

Since the hyperbolic metric $\rho_{a}$ is equivalent to a metric of $X$ with the same topology as long as $a^{\prime}<\sqrt{2}-1$ [16], we always take $\rho_{a}$ as a metric for simplicity. Under this metric we then can complete the space $X$ and denote by the completion $\hat{X}$. We call $\partial X:=\hat{X} \backslash X$ the hyperbolic boundary of $X$. The metric $\rho_{a}$ can be extended onto $\partial X$, and under which $\partial X$ is a compact set. It is often useful to identify $\xi \in \partial X$ with the geodesic rays in $X$ that converge to $\xi$, i.e., an infinite path $\pi\left[x_{1}, x_{2}, \ldots\right]$ such that $x_{i} \sim x_{i+1}$ and any finite segment of the path is a geodesic. It is known that two geodesic rays $\pi\left[x_{1}, x_{2}, \ldots\right], \pi\left[y_{1}, y_{2}, \ldots\right]$ represent the same $\xi \in \partial X$ if and only if $\left|x_{n} \wedge y_{n}\right| \rightarrow \infty$ as $n \rightarrow \infty$.

Our interest is on the following tree structure introduced by Kamainovich which is used to study the self-similar sets $[6,10]$. For a tree $X$ with a root $o$, we use $\mathcal{E}_{v}$ to denote the set of edges ( $v$ for vertical). We introduce additional edges on each level $\{x: d(0, x)=n\}, n \in \mathbf{N}$ as follows. Let $x^{-k}$ denote the $k$-th ancestor of $x$, the unique point in $(n-k)$-th level that is joined by a unique path.

Definition 2.1. [6] Let $X$ be a tree with a root $o$. Let $\mathcal{E}_{h} \subset(X \times X) \backslash\{(x, x): x \in$ $X\}$ such that it is symmetric and satisfies:

$$
(x, y) \in \mathcal{E}_{h} \quad \Rightarrow \quad|x|=|y| \text { and either } x^{-1}=y^{-1} \text { or }\left(x^{-1}, y^{-1}\right) \in \mathcal{E}_{h} .
$$

We call elements in $\mathcal{E}_{h}$ horizontal edges, and for $\mathcal{E}=\mathcal{E}_{v} \cup \mathcal{E}_{h},(X, \mathcal{E})$ is called an augmented tree.

Following from [10], we say that a path $\pi(x, y)$ is a horizontal geodesic if it is a geodesic and consisting of horizontal edges only. A path is called a canonical geodesic if there exist $u, v \in \pi(x, y)$ such that:

(i) $\pi(x, y)=\pi(x, u) \cup \pi(u, v) \cup \pi(v, y)$ with $\pi(u, v)$ a horizontal path and $\pi(x, u)$, $\pi(v, y)$ vertical paths;

(ii) for any geodesic path $\pi^{\prime}(x, y)$, $\operatorname{dist}(o, \pi(x, y)) \leq \operatorname{dist}\left(o, \pi^{\prime}(x, y)\right)$.

Note that condition (ii) is to require the horizontal part of the canonical geodesic to be on the highest level. The following theorem is due to Lau and Wang [10].

Theorem 2.2. Let $X$ be an augmented tree. Then

(i) Let $\pi(x, y)$ be a canonical geodesic, then $|x \wedge y|=l-h / 2$, where $l$ and $h$ are respectively the level and length of the horizontal part of the geodesic.

(ii) $X$ is hyperbolic if and only if the lengths of horizontal geodesics are uniformly bounded.

The major application of the augmented trees is to identify their boundaries with the self-similar sets. We assume a self-similar set $K$ is generated by an iterated function system (IFS) $\left\{S_{j}\right\}_{j=1}^{m}$ on $\mathbf{R}^{d}$. Let $X=\bigcup_{n=0}^{\infty}\{1, \ldots, m\}^{n}$ be the symbolic space representing the IFS (by convention, $\{1, \ldots, m\}^{0}=\emptyset$, and we still denote it by $o)$. For $\mathbf{u}=i_{1} \cdots i_{k}$, we denote by $S_{\mathbf{u}}=S_{i_{1}} \circ \cdots \circ S_{i_{k}}$. Let $\mathcal{E}_{v}$ be the set of vertical edges corresponding to the nature tree structure on $X$ with $\emptyset$ as a root. We define 
the horizontal edges on $X$ by

$$
\mathcal{E}_{h}=\left\{(\mathbf{u}, \mathbf{v}):|\mathbf{u}|=|\mathbf{v}|, \mathbf{u} \neq \mathbf{v} \text { and } K_{\mathbf{u}} \cap K_{\mathbf{v}} \neq \emptyset\right\},
$$

where $K_{\mathbf{u}}=S_{\mathbf{u}}(K)$. Let $\mathcal{E}=\mathcal{E}_{v} \cup \mathcal{E}_{h}$, then $(X, \mathcal{E})$ is an augmented tree induced by the self-similar set.

It was proved in [10] that under the open set condition (OSC), the augmented tree is hyperbolic and its hyperbolic boundary can be identified with the self-similar set. More generally, Wang [14] extended these results to the weak separation condition (WSC), the definition was introduced by Lau and Ngai [9] to study the multifractal structure for an IFS with overlaps.

In [8], we have discussed that the choice of the horizontal edges for the augmented tree is quite flexible. Sometimes, we prefer to use another setting by replacing $K$ with a bounded closed invariant set $J$ (i.e., $S_{i}(J) \subset J$ for each $i$ ), namely

$$
\mathcal{E}_{h}=\left\{(\mathbf{u}, \mathbf{v}):|\mathbf{u}|=|\mathbf{v}|, \mathbf{u} \neq \mathbf{v} \text { and } J_{\mathbf{u}} \cap J_{\mathbf{v}} \neq \emptyset\right\}
$$

where $J_{\mathbf{u}}:=S_{\mathbf{u}}(J)$. We can take $J=K$ as before or in many situations, take $J=\bar{U}$ for the $U$ in the OSC. The hyperbolicity and identity still hold by adopting the same proof.

We remark that the augmented tree $(X, \mathcal{E})$ depends on the choice of the bounded invariant set $J$. But under the OSC or WSC, the hyperbolic boundary is the same as they can be identified with the underlying self-similar set. In the following section, we will discuss the augmented tree and its hyperbolic structure induced by a Moran set.

\section{Moran sets as hyperbolic boundaries}

Recall that $\mathcal{M}:=\mathcal{M}\left(J,\left\{n_{k}\right\},\left\{r_{k}\right\}\right)$ denotes the class of the Moran sets satisfying (i)-(iv) of Definition 1.1 and $r:=\inf _{k} r_{k}>0$.

Lemma 3.1. Suppose $E \in \mathcal{M}$ is a Moran set. Then for any $b>0$, there exists a constant $c>0$ (depending on $b$ ) such that for any $n$ and a set $V \subset \mathbf{R}^{d}$ with diameter $|V| \leq b r^{n}$, we have

$$
\#\left\{\mathbf{i} \in X_{n}: J_{\mathbf{i}} \cap V \neq \emptyset\right\} \leq c .
$$

Proof. The proof is the same as the case for a self-similar set satisfying OSC by applying a geometrical lemma (see Lemma 9.2 of Falconer [1]).

We now construct a graph on $X=\bigcup_{n>0} X_{n}$. For each word $\mathbf{u} \in X_{n}$, there exists a unique word $\mathbf{v} \in X_{n-1}$ and a finite word $\mathbf{v}^{\prime}$ such that $\mathbf{u}=\mathbf{v v}^{\prime}$. We denote the unique $\mathbf{v}$ by $\mathbf{u}^{-1}$ and call it the first ancestor of $\mathbf{u}$, inductively $\mathbf{u}^{-k}=\left(\mathbf{u}^{-(k-1)}\right)^{-1}$. It follows that $\mathbf{u}, \mathbf{u}^{-1}, \ldots, \mathbf{u}^{-k}$ is a path from $\mathbf{u}^{-k}$ to $\mathbf{u}$. The natural tree structure on $X$ is to take $\emptyset$ as the root $O$ and to define the set of vertical edges in this way

$$
\mathcal{E}_{v}=\left\{\left(\mathbf{u}^{-1}, \mathbf{u}\right): \mathbf{u} \in X \backslash\{\emptyset\}\right\} .
$$

The horizontal edge set is defined by

$$
\mathcal{E}_{h}=\left\{(\mathbf{u}, \mathbf{v}): \exists n>0 \text { such that } \mathbf{u} \neq \mathbf{v} \in X_{n} \text { and } J_{\mathbf{u}} \cap J_{\mathbf{v}} \neq \emptyset\right\} .
$$

Let $\mathcal{E}=\mathcal{E}_{v} \cup \mathcal{E}_{h}$. With the analogous argument in the proof of Theorem 3.2 of [10], it concludes that

Proposition 3.2. Suppose $E \in \mathcal{M}$ is a Moran set. Then the induced augmented tree $(X, \mathcal{E})$ is a hyperbolic graph. 
Proof. First we show the graph $X$ is locally finite. By Lemma 3.1, there exists $c>0$ such that for any $n>0$ and $\mathbf{v} \in X_{n}$,

$$
\#\left\{\mathbf{u} \in X_{n}: J_{\mathbf{u}} \cap J_{\mathbf{v}} \neq \emptyset\right\} \leq c .
$$

Therefore $\mathbf{v}$ has at most $c$ neighbors in the same level, also it has one ancestor. On the other hand, let $\mathbf{u}$ be a descendant of $\mathbf{v}$, i.e., $\mathbf{u}^{-1}=\mathbf{v}$, then $J_{\mathbf{u}} \subset J_{\mathbf{v}}$, hence $J_{\mathbf{u}} \cap J_{\mathbf{v}} \neq \emptyset$. Let $V=J_{\mathbf{v}}$, by using Lemma 3.1 again, we obtain

$$
\#\left\{\mathbf{u} \in X: \mathbf{u}^{-1}=\mathbf{v}\right\} \leq c^{\prime} .
$$

Hence for $\mathbf{v} \in X$,

$$
\operatorname{deg}(\mathbf{v})=\#\{(\mathbf{u}, \mathbf{v}) \in \mathcal{E}: \mathbf{u} \in X\} \leq c+c^{\prime}+1,
$$

and $X$ is locally finite.

Next we show that the lengths of the horizontal geodesics are uniformly bounded, then Theorem 2.2 implies that $(X, \mathcal{E})$ is hyperbolic. Suppose otherwise, for any integer $k>0$, there exists a horizontal geodesic $\pi\left(\mathbf{u}_{0}, \mathbf{u}_{3 k}\right)=\left[\mathbf{u}_{0}, \mathbf{u}_{1}, \ldots, \mathbf{u}_{3 k}\right]$ with $\mathbf{u}_{i} \in X_{n}$. We consider the $k$-th ancestors $\mathbf{v}_{i}=\mathbf{u}_{i}^{-k}$ and the path $\left[\mathbf{v}_{0}, \mathbf{v}_{1}, \ldots, \mathbf{v}_{3 k}\right]=$ $\left[\mathbf{u}_{0}^{-k}, \mathbf{u}_{1}^{-k}, \ldots, \mathbf{u}_{3 k}^{-k}\right]$. Let

$$
p\left(\mathbf{v}_{0}, \mathbf{v}_{3 k}\right)=\left[\mathbf{v}_{i_{0}}, \mathbf{v}_{i_{1}}, \ldots, \mathbf{v}_{i_{\ell}}\right], \quad \mathbf{v}_{i_{j}} \in\left\{\mathbf{v}_{0}, \ldots, \mathbf{v}_{3 k}\right\},
$$

be the shortest horizontal path connecting $\mathbf{v}_{0}$ and $\mathbf{v}_{3 k}$. By the geodesic property of $\pi\left(\mathbf{u}_{0}, \mathbf{u}_{3 k}\right)$, it is clear that

$$
\ell=\left|p\left(\mathbf{v}_{0}, \mathbf{v}_{3 k}\right)\right| \geq\left|\pi\left(\mathbf{u}_{0}, \mathbf{u}_{3 k}\right)\right|-2 k=k .
$$

Now choose $k \geq c$ such that $(3 k+1) r^{k} \leq 1$, where $c$ is as in Lemma 3.1. Let

$$
V=\bigcup_{i=0}^{3 k} J_{\mathbf{u}_{i}} .
$$

From $\left|J_{\mathbf{u}_{i}}\right| \leq r^{n}|J|, i=0,1, \ldots, 3 k$, it is straightforward to show that

$$
|V| \leq(3 k+1) r^{n}|J| \leq r^{n-k}|J| .
$$

Note that $J_{\mathbf{u}_{i}} \subset J_{\mathbf{v}_{i}}$, we see that $J_{\mathbf{v}_{i_{j}}} \cap V \neq \emptyset$ for each $j=0,1, \ldots, \ell$. It follows that

$$
\#\left\{\mathbf{v} \in X_{n-k}: J_{\mathbf{v}} \cap V \neq \emptyset\right\} \geq \ell+1>k \geq c .
$$

That contradicts Lemma 3.1 and the proof is complete.

Remember that the hyperbolic boundary $\partial X$ is a compact set under the metric $\rho_{a}$ and any element $\xi \in \partial X$ is often identified as an equivalence class of geodesic rays $\pi\left[\mathbf{u}_{1}, \mathbf{u}_{2}, \ldots\right]$ in $X$. Now we give the main theorem of this section which extends the results on self-similar sets of Lau and Wang [10] to that on Moran sets. First a condition $(\mathrm{H})$ is needed.

Condition (H): There is a positive constant $C^{\prime}$ such that for any $n \geq 1$ and $\mathbf{u}, \mathbf{v} \in X_{n}$, either $J_{\mathbf{u}} \cap J_{\mathbf{v}} \neq \emptyset$ or $\operatorname{dist}\left(J_{\mathbf{u}}, J_{\mathbf{v}}\right) \geq C^{\prime} r^{n}$.

There are many standard self-similar sets satisfying condition $(\mathrm{H})$, for example, the generating IFS has the OSC and all the parameters of the similitudes are integers. However there are also examples that condition $(\mathrm{H})$ is not satisfied (see [10, 14]). 
Theorem 3.3. Let $E \in \mathcal{M}$ be a Moran set, $(X, \mathcal{E})$ be the induced augmented tree. Then there exists a bijection $\Phi: \partial X \rightarrow E$ satisfying:

$$
|\Phi(\xi)-\Phi(\eta)| \leq C \rho_{a}(\xi, \eta)^{\alpha}, \quad \text { for any } \xi, \eta \in \partial X,
$$

where $\alpha=-\log r / a$. In this case $\partial X$ is homeomorphic to $E$.

If the additional condition $(H)$ holds, then this $\Phi$ has the following Hölder equivalent property:

$$
C^{-1}|\Phi(\xi)-\Phi(\eta)| \leq \rho_{a}(\xi, \eta)^{\alpha} \leq C|\Phi(\xi)-\Phi(\eta)| .
$$

Proof. Let $\mathbf{u}_{0}=\emptyset$, for any geodesic ray $\xi=\pi\left[\mathbf{u}_{1}, \mathbf{u}_{2}, \ldots\right]$, define

$$
\Phi(\xi)=\lim _{n \rightarrow \infty} S_{\mathbf{u}_{n}}\left(x_{0}\right)
$$

for some $x_{0} \in J$. It is easy to show the mapping is well-defined. Indeed, if two geodesic rays $\xi=\pi\left[\mathbf{u}_{0}, \mathbf{u}_{1}, \ldots\right], \eta=\pi\left[\mathbf{v}_{0}, \mathbf{v}_{1}, \ldots\right]$ are equivalent, then there exists a constant $c>0$ such that

$$
d\left(\mathbf{u}_{n}, \mathbf{v}_{n}\right) \leq c \delta
$$

for all $n \geq 0$, where $\delta>0$ depends only on the hyperbolicity of the graph $X$ [16]. Let $\mathbf{u}_{n}=\mathbf{t}_{0}, \mathbf{t}_{1}, \ldots, \mathbf{t}_{k}=\mathbf{v}_{n}$ be a canonical geodesic from $\mathbf{u}_{n}$ to $\mathbf{v}_{n}$, then $k \leq c \delta$. The canonical geodesic can be written in three parts, two vertical and one horizontal parts: $\mathbf{t}_{0}, \ldots, \mathbf{t}_{i} ; \mathbf{t}_{i}, \ldots, \mathbf{t}_{j}$ and $\mathbf{t}_{j}, \ldots, \mathbf{t}_{k}$. For the horizontal part, we assume that $\mathbf{t}_{i}, \ldots, \mathbf{t}_{j} \in X_{\ell_{n}}$, i.e. the $\ell_{n}$-th level. Note that

$$
J_{\mathbf{t}_{0}} \subset J_{\mathbf{t}_{1}} \subset \cdots \subset J_{\mathbf{t}_{i}} \text { and } J_{\mathbf{t}_{j}} \supset J_{\mathbf{t}_{j-1}} \supset \cdots \supset J_{\mathbf{t}_{k}} .
$$

Then we have

and

$$
\left|S_{\mathbf{u}_{n}}\left(x_{0}\right)-S_{\mathbf{t}_{i}}\left(x_{0}\right)\right| \leq\left|J_{\mathbf{t}_{i}}\right| \leq r^{\ell_{n}}|J|
$$

$$
\left|S_{\mathbf{v}_{n}}\left(x_{0}\right)-S_{\mathbf{t}_{j}}\left(x_{0}\right)\right| \leq\left|J_{\mathbf{t}_{j}}\right| \leq r^{\ell_{n}}|J|
$$

Since the horizontal part $\left[\mathbf{t}_{i}, \ldots, \mathbf{t}_{j}\right]$ lies in the same horizontal level $\ell_{n}$, it follows that $r_{\mathbf{t}_{i}}, \ldots, r_{\mathbf{t}_{j}} \leq r^{\ell_{n}}$ and $j-i \leq c \delta$, hence

$$
\left|S_{\mathbf{t}_{i}}\left(x_{0}\right)-S_{\mathbf{t}_{j}}\left(x_{0}\right)\right| \leq(j-i+1) r^{\ell_{n}}|J| \leq(c \delta+1) r^{\ell_{n}}|J| .
$$

Combining the above estimates together, we conclude

$$
\left|S_{\mathbf{u}_{n}}\left(x_{0}\right)-S_{\mathbf{v}_{n}}\left(x_{0}\right)\right| \leq C r^{\ell_{n}}
$$

for some constant $C>0$. Obverse that $\lim _{n \rightarrow \infty} \ell_{n}=\lim _{n \rightarrow \infty}\left|\mathbf{u}_{n} \wedge \mathbf{v}_{n}\right|=+\infty$. Consequently, $\lim _{n \rightarrow \infty} S_{\mathbf{u}_{n}}\left(x_{0}\right)=\lim _{n \rightarrow \infty} S_{\mathbf{v}_{n}}\left(x_{0}\right)$ and $\Phi$ is well-defined.

For any $x \in E$, there exists an infinite word $i_{1} i_{2} \cdots \in D_{\infty}$ such that

$$
\lim _{n \rightarrow \infty} S_{i_{1} i_{2} \cdots i_{n}}\left(x_{0}\right)=x .
$$

Let $\mathbf{u}_{0}=\emptyset$ and for every $n \geq 1$, there exists a unique $k_{n}$ such that $r_{1} \cdots r_{k_{n}} \leq r^{n}<$ $r_{1} \cdots r_{k_{n}-1}$, denote $\mathbf{u}_{n}=i_{1} \cdots i_{k_{n}}$ and $\xi=\pi\left[\mathbf{u}_{0}, \mathbf{u}_{1}, \ldots\right]$. Then $\xi \in \partial X$ and $\Phi(\xi)=x$ which proved that $\Phi$ is surjective. If $\Phi(\xi)=\Phi(\eta)=x \in E$, then $x \in J_{\mathbf{u}_{n}} \cap J_{\mathbf{v}_{n}}$ for $n \geq 0$, hence $d\left(\mathbf{u}_{n}, \mathbf{v}_{n}\right) \leq 1$, and $\xi, \eta$ are equivalent. Whence $\Phi$ is bijective.

In the following, we show that $\Phi$ is Hölder continuous. Then being a bijective continuous map, $\Phi$ is a homeomorphism. Let $\xi=\pi\left[\mathbf{u}_{0}, \mathbf{u}_{1}, \mathbf{u}_{2}, \ldots\right], \eta=\pi\left[\mathbf{v}_{0}, \mathbf{v}_{1}, \mathbf{v}_{2}, \ldots\right]$ be any two non-equivalent geodesic rays in $X$. Then there is a bilateral geodesic $\gamma$ joining $\xi$ and $\eta[16]$. We assume that it is canonical:

$$
\gamma=\pi\left[\ldots, \mathbf{u}_{n+1}, \mathbf{u}_{n}, \mathbf{t}_{1}, \ldots, \mathbf{t}_{\ell}, \mathbf{v}_{n}, \mathbf{v}_{n+1}, \ldots\right]
$$


with $\mathbf{u}_{n}, \mathbf{t}_{1}, \ldots, \mathbf{t}_{\ell}, \mathbf{v}_{n} \in X_{n}$, i.e. $n$-th level. It follows that

$$
\left|S_{\mathbf{u}_{n}}\left(x_{0}\right)-S_{\mathbf{v}_{n}}\left(x_{0}\right)\right| \leq(\ell+2) r^{n}|J| .
$$

By the hyperbolicity of the augmented tree $X, \ell$ is uniformly bounded by a constant which depends only on the graph. Since $\Phi(\xi) \in J_{\mathbf{u}_{k}}$ and $\Phi(\eta) \in J_{\mathbf{v}_{k}}$ for all $k \geq 0$, we get

$$
\left|\Phi(\xi)-S_{\mathbf{u}_{n}}\left(x_{0}\right)\right|, \quad\left|\Phi(\eta)-S_{\mathbf{v}_{n}}\left(x_{0}\right)\right| \leq r^{n}|J|
$$

Thus

$$
|\Phi(\xi)-\Phi(\eta)| \leq\left|\Phi(\xi)-S_{\mathbf{u}_{n}}\left(x_{0}\right)\right|+\left|S_{\mathbf{u}_{n}}\left(x_{0}\right)-S_{\mathbf{v}_{n}}\left(x_{0}\right)\right|+\left|\Phi(\eta)-S_{\mathbf{v}_{n}}\left(x_{0}\right)\right| \leq C_{1} r^{n} .
$$

Since it is a bilateral canonical geodesic, we have $|\xi \wedge \eta|=n-(\ell+1) / 2$ and $\ell$ is uniformly bounded. By using $\rho_{a}(\xi, \eta)=\exp (-a|\xi \wedge \eta|)$, it yields that

$$
|\Phi(\xi)-\Phi(\eta)| \leq C \rho_{a}(\xi, \eta)^{\alpha} .
$$

For the second part, if the additional condition holds, assume that $\xi \neq \eta$. Since $\gamma$ in (3.2) is a geodesic, it follows that $\left(\mathbf{u}_{n+1}, \mathbf{v}_{n+1}\right) \notin \mathcal{E}_{h}$, and hence $J_{\mathbf{u}_{n+1}} \cap J_{\mathbf{v}_{n+1}}=\emptyset$ which implies

$$
|\Phi(\xi)-\Phi(\eta)| \geq \operatorname{dist}\left(J_{\mathbf{u}_{n+1}}, J_{\mathbf{v}_{n+1}}\right) \geq C^{\prime} r^{n+1},
$$

and the theorem follows in view of the definition of the metric $\rho_{a}$.

\section{Lipschitz equivalence}

Two compact metric spaces $\left(X, d_{X}\right)$ and $\left(Y, d_{Y}\right)$ are said to be Lipschitz equivalent, and denote by $X \simeq Y$, if there is a bi-Lipschitz map $\sigma$ from $X$ onto $Y$, i.e., $\sigma$ is a bijection and there is a constant $C>0$ such that

$$
C^{-1} d_{X}(x, y) \leq d_{Y}(\sigma(x), \sigma(y)) \leq C d_{X}(x, y) \quad \forall x, y \in X .
$$

Following [8], we have

Definition 4.1. Let $X$ and $Y$ be two hyperbolic graphs and let $\sigma: X \rightarrow Y$ be a bijective map. We say that $\sigma$ is a near-isometry if there exists $c>0$ such that

$$
|| \pi(\sigma(x), \sigma(y))|-| \pi(x, y)|| \leq c \quad \forall x, y \in X .
$$

Proposition 4.2. ([8]) Let $X, Y$ be two hyperbolic augmented trees that are equipped with the hyperbolic metrics with the same parameter a (as in (2.1)). Suppose there exists a near-isometry $\sigma: X \rightarrow Y$, then $\partial X \simeq \partial Y$.

According to conditions (iii) and (iv) of Definition 1.1, it follows that every basic set $J_{\mathbf{i}}$ (say $\mathbf{i} \in D_{k-1}$ ) gives rise to the same number of subsets (say $n_{k}$ ) under the same contraction ratio (say $r_{k}$ ) from one step to the next. That, together with (1.1), implies that, for an augmented tree $X=\bigcup_{n>0} X_{n}$, any two words of the level $X_{n}$ generate the same number of offsprings in the level $X_{n+1}$.

By a horizontal connected components of an augmented tree $X$, we mean a maximal connected horizontal subgraph on some level $X_{n}$. Let $\mathscr{C}$ be the set of all horizontal connected components of $X$. For $T \in \mathscr{C}$, say, it lies in the level $X_{n}$, we let

$$
T \Sigma_{n}=\left\{\mathbf{u i} \in X_{n+1}: \mathbf{u} \in T, \mathbf{i} \in \Sigma_{n}\right\}
$$

denote the set of offsprings of $T$ in the level $X_{n+1}$, where $\Sigma_{n}$ denotes the suffix set of words. By the previous argument, we note that the $\Sigma_{n}$ only depends on the level $X_{n}$, 
i.e., $\Sigma_{n}=\prod_{j=a(n)}^{b(n)}\left\{1,2, \ldots, n_{j}\right\}$ where $a(n) \leq b(n)$ are positive integers depending on $n$ only. If no confusion occurs, we write $\Sigma:=\Sigma_{n}$ for simplicity.

With the above notation, we introduce a key concept of this section.

Definition 4.3. An augmented tree $X$ is called rearrangeable if $\max \{\# T: T \in$ $\mathscr{C}\}<\infty$, and for any $T \in \mathscr{C}$ with $\# T=b$, its offsprings $T \Sigma$ of the next level can be decomposed into $b$ groups as following

$$
T \Sigma=\left(\bigcup_{k \in \Lambda_{1}} Z_{k}\right) \cup \cdots \cup\left(\bigcup_{k \in \Lambda_{b}} Z_{k}\right)
$$

such that every $Z_{k} \in \mathscr{C}$ consists of the offsprings of $T$ and the total size of every group is equal to $\# \Sigma$.

In fact, the concept of 'rearrangeable augment tree' coincides with the two additional conditions (v) and (vi) of Moran sets defined in the introduction. Recall that $\mathcal{M}^{\prime}:=\mathcal{M}\left(J,\left\{n_{k}\right\}, r\right)$ is the collection of all Moran sets satisfying conditions (v) and (vi). It is easy to verify that if a Moran set $E \in \mathcal{M}^{\prime}$ then the induced augmented tree $X$ is rearrangeable. Moreover, if we assume $n_{k} \equiv n$ and $r_{k} \equiv r$ and choose a fixed IFS in Definition 1.1, then the Moran set degenerates to the self-similar set with equal ratio and the discussion of this section goes back to the case considered by [8].

Theorem 4.4. Suppose the augmented tree $(X, \mathcal{E})$ is rearrangeable. Then there is a near-isometry between $(X, \mathcal{E})$ and $\left(X, \mathcal{E}_{v}\right)$ so that $(X, \mathcal{E}) \simeq\left(X, \mathcal{E}_{v}\right)$.

Proof. Let $X=(X, \mathcal{E}), Y=\left(X, \mathcal{E}_{v}\right)$. It suffices to construct a near-isometry $\sigma$ between $X$ and $Y$, and hence $\partial(X, \mathcal{E}) \simeq \partial\left(X, \mathcal{E}_{v}\right)$ by Proposition 4.2. We define this $\sigma$ to be a one-to-one mapping from $X_{n}$ (in $X$ ) to $X_{n}$ (in $Y$ ) inductively as follows: Let

$$
\sigma(o)=o, \quad \text { and } \quad \sigma(x)=x, x \in X_{1} .
$$

Suppose $\sigma$ is defined on the level $n$ (i.e., $X_{n}$ ) such that for every horizontal connected component $T, \sigma(T)$ has the same parent, i.e.,

$$
\sigma(x)^{-1}=\sigma(y)^{-1} \quad \forall x, y \in T \subset X_{n}
$$

(see Figure 1).

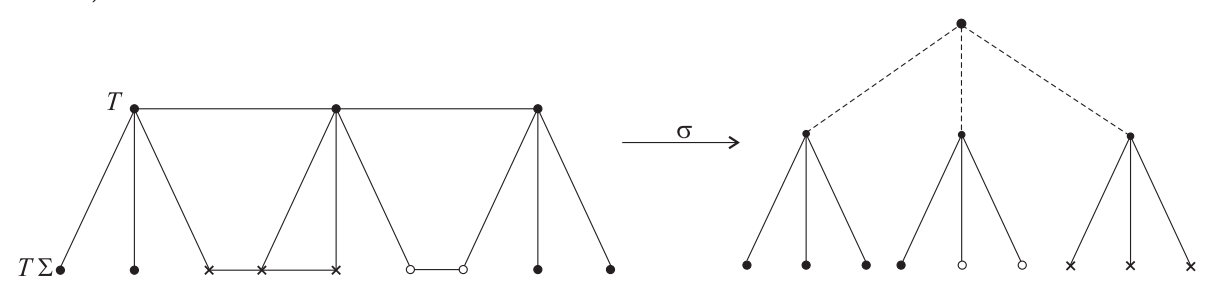

Figure 1. An illustration of the rearrangeable condition by $\sigma$.

To define the map $\sigma$ on $X_{n+1}$, we note that $T$ in $X_{n}$ gives rise to horizontal connected components in $X_{n+1}$. We can write

$$
T \Sigma=\bigcup_{k=1}^{\ell} Z_{k}
$$

where $\Sigma$ is the set of suffixes such that $T \Sigma \subset X_{n+1}$, and $Z_{k}$ are horizontal connected components consisting of offsprings of $T$. Let $\# T=b$ and $\# \Sigma=m$, by the rearrangeable condition, $\bigcup_{k=1}^{\ell} Z_{k}$ can be rearranged as $b$ groups so that total size of every 
group is equal to $m$, namely,

$$
\bigcup_{k=1}^{\ell} Z_{k}=\bigcup_{k \in \Lambda_{1}} Z_{k} \cup \cdots \cup \bigcup_{k \in \Lambda_{b}} Z_{k}
$$

Note that each set on the right has $m$ elements.

For the connected component $T=\left\{\mathbf{i}_{1}, \ldots, \mathbf{i}_{b}\right\} \subset X_{n}$, we have defined $\sigma$ on $X_{n}$ and $\sigma(T)=\left\{\mathbf{j}_{1}=\sigma\left(\mathbf{i}_{1}\right), \ldots, \mathbf{j}_{b}=\sigma\left(\mathbf{i}_{b}\right)\right\}$. In view of (4.2), we define $\sigma$ on $T \Sigma=\bigcup_{k=1}^{\ell} Z_{k}$ by assigning each $\bigcup_{k \in \Lambda_{s}} Z_{k}$ (it has $m$ elements) the $m$ descendants of $\mathbf{j}_{s}$ (see Figure 1 ). It is clear that $\sigma$ is well-defined on $T \Sigma$ and satisfies (4.1) for $x, y \in T \Sigma$. We apply the same construction of $\sigma$ on the offsprings of every horizontal connected component in $X_{n}$. It follows that $\sigma$ is well-defined and satisfies (4.1) on $X_{n+1}$. Inductively, $\sigma$ can be defined from $X$ to $Y$ and is bijective.

Finally we show that $\sigma$ is indeed a near-isometry and complete the proof. Since $\sigma: X \rightarrow Y$ preserves the levels, hence without loss of generality, it suffices to prove the near-isometry for $\mathbf{x}, \mathbf{y}$ belong to the same level. Let $\pi(\mathbf{x}, \mathbf{y})$ be the canonical geodesic connecting them, which can be written as

$$
\pi(\mathbf{x}, \mathbf{y})=\left[\mathbf{x}, \mathbf{u}_{1}, \ldots, \mathbf{u}_{n}, \mathbf{t}_{1}, \ldots, \mathbf{t}_{k}, \mathbf{v}_{n}, \ldots, \mathbf{v}_{1}, \mathbf{y}\right]
$$

where $\left[\mathbf{t}_{1}, \ldots, \mathbf{t}_{k}\right]$ is the horizontal part and $\left[\mathbf{x}, \mathbf{u}_{1}, \ldots, \mathbf{u}_{n}, \mathbf{t}_{1}\right],\left[\mathbf{t}_{k}, \mathbf{v}_{n}, \ldots, \mathbf{v}_{1}, \mathbf{y}\right]$ are vertical parts. Clearly, $\left\{\mathbf{t}_{1}, \ldots, \mathbf{t}_{k}\right\}$ must be included in one horizontal connected component of $X$, we denote it by $T^{\prime}$. With the notation as in Theorem 2.2(i), it follows that for $\mathbf{x} \neq \mathbf{y} \in X$,

$$
|\pi(\mathbf{x}, \mathbf{y})|=|\mathbf{x}|+|\mathbf{y}|-2 l+h, \quad|\pi(\sigma(\mathbf{x}), \sigma(\mathbf{y}))|=|\sigma(\mathbf{x})|+|\sigma(\mathbf{y})|-2 l^{\prime}+h^{\prime} .
$$

We have

$$
|| \pi(\sigma(\mathbf{x}), \sigma(\mathbf{y}))|-| \pi(\mathbf{x}, \mathbf{y})|| \leq\left|h-h^{\prime}\right|+2\left|l^{\prime}-l\right| \leq k+2\left|l^{\prime}-l\right|
$$

where $k$ is a hyperbolic constant as in Theorem 2.2(ii). If $T^{\prime}$ is a singleton, then

$$
\left|l^{\prime}-l\right|=0 \text {. }
$$

If $T^{\prime}$ contains more than one point, then the elements of $\sigma\left(T^{\prime}\right)$ share the same parent. Then the confluence of $\sigma(\mathbf{x})$ and $\sigma(\mathbf{y})$ (as a tree) is $\sigma(\mathbf{x})^{-1}\left(=\sigma(\mathbf{y})^{-1}\right)$. Hence

$$
\left|l^{\prime}-l\right|=1
$$

Consequently,

$$
|| \pi(\sigma(\mathbf{x}), \sigma(\mathbf{y}))|-| \pi(\mathbf{x}, \mathbf{y})|| \leq k+2 .
$$

This completes the proof that $\sigma$ is a near-isometry and the theorem is established.

Corollary 4.5. Under the assumption on the above theorem, then $\left(\partial(X, \mathcal{E}), \rho_{a}\right)$ is totally disconnected.

By Theorem 4.4, we obtain the following Lipschitz equivalence on Moran sets. Furthermore, Theorem 1.3 can be proved as well.

Theorem 4.6. Let $E, E^{\prime} \in \mathcal{M}$ be two Moran sets, and satisfy condition (H). Assume the associated augmented trees both are rearrangeable. Then $E$ and $E^{\prime}$ are Lipschitz equivalent.

Proof. It follows from Theorem 4.4 that

$$
\partial(X, \mathcal{E}) \simeq \partial\left(X, \mathcal{E}_{v}\right)=\partial\left(Y, \mathcal{E}_{v}\right) \simeq \partial(Y, \mathcal{E})
$$


(for the respective metrics $\rho_{a}$ ). Let $\varphi: \partial(X, \mathcal{E}) \rightarrow \partial(Y, \mathcal{E})$ be the bi-Lipschitz map. With no confusion, we just denote these two boundaries by $\partial X, \partial Y$ as before.

By Theorem 3.3, there exist two bijections $\Phi_{1}: \partial X \rightarrow E$ and $\Phi_{2}: \partial Y \rightarrow E^{\prime}$ satisfying (3.1) with constants $C_{1}, C_{2}$, respectively. Define $\tau: E \rightarrow E^{\prime}$ as

$$
\tau=\Phi_{2} \circ \varphi \circ \Phi_{1}^{-1} \text {. }
$$

Then

$$
\begin{aligned}
|\tau(x)-\tau(y)| & \leq C_{2} \rho_{a}\left(\varphi \circ \Phi_{1}^{-1}(x), \varphi \circ \Phi_{1}^{-1}(y)\right)^{\alpha} \\
& \leq C_{2} C_{0}^{\alpha} \rho_{a}\left(\Phi_{1}^{-1}(x), \Phi_{1}^{-1}(y)\right)^{\alpha} \leq C_{2} C_{0}^{\alpha} C_{1}|x-y| .
\end{aligned}
$$

Let $C^{\prime}=C_{2} C_{0}^{\alpha} C_{1}$, then

$$
|\tau(x)-\tau(y)| \leq C^{\prime}|x-y|
$$

Similarly, we have $C^{\prime-1}|x-y| \leq|\tau(x)-\tau(y)|$. Therefore $\tau: E \rightarrow E^{\prime}$ is a bi-Lipschitz map.

Under the Hölder equivalent property (3.1), the proof of the above theorem still yields an interesting result.

Corollary 4.7. Let $E, E^{\prime} \in \mathcal{M}$ be two Moran sets satisfying condition (H), let $\partial X$ and $\partial Y$ be their hyperbolic boundaries, respectively. Then

$$
E \simeq E^{\prime} \Leftrightarrow \partial X \simeq \partial Y .
$$

Acknowledgements. The author would like to thank Professor Ka-Sing Lau for many valuable comments.

\section{References}

[1] FAlconer, K. J.: Fractal geometry, mathematical foundation and applications. - John Wiley \& Sons, New York, 1990.

[2] Feng, D. J., Z. Y. Wen, and J. Wu: Some dimensional results for homogeneous Moran sets. - Sci. China Ser. A 40:5, 1997, 475-482.

[3] Gromov, M.: Hyperbolic groups. - In: Essays in group theory, Math. Sci. Res. Inst. Publ. 8, Springer, 1987, 75-263.

[4] Hua, S., H. RaO, Z. Y. Wen, and J. Wu: On the structures and dimensions of Moran sets. - Sci. China Ser. A 43:8, 2000, 836-852.

[5] Ju, H. B., K. S. LAu, and X. Y. Wang: Post-critically finite fractal and Martin boundary. Trans. Amer. Math. Soc. 364:1, 2012, 103-118.

[6] Kaimanovich, V.A.: Random walks on Sierpinski graphs: Hyperbolicity and stochastic homogenization. - In: Fractals in Graz 2001, Trends Math., Birkhäuser, Basel, 2003, 145-183.

[7] Kigami, J.: Dirichlet forms and associated heat kernels on the Cantor set induced by random walks on trees. - Adv. Math. 225:5, 2010, 2674-2730.

[8] LAU, K. S., and J. J. LUO: Lipschitz equivalence of self-similar sets and hyperbolic boundaries. - Preprint.

[9] LAU, K. S., and S. M. NGAI: Multifractal measures and a weak separation condition. - Adv. Math. 141, 1999, 45-96.

[10] LAU, K. S., and X. Y. WANG: Self-similar sets as hyperbolic boundaries. - Indiana Univ. Math. J. 58, 2009, 1777-1795.

[11] LAU, K. S., and X. Y. WANG: Self-similar sets, hyperbolic boundaries and Martin boundaries. - Preprint. 
[12] Luo, J. J.: Topological structure and Lipschitz equivalence of fractal sets. - Ph.D. Thesis, The Chinese University of Hong Kong, 2012.

[13] Moran, P. A. P.: Additive functions of intervals and Hausdorff measure. - Proc. Cambridge Philos. Soc. 42, 1946, 15-23.

[14] Wang, X. Y.: Graphs induced by iterated function systems. - Preprint.

[15] Wen, Z. Y.: Moran sets and Moran classes. - China Sci. Bull. 46:22, 2001, 1849-1856.

[16] Woess, W.: Random walks on infinite graphs and groups. - Cambridge Tracts in Math. 138, Cambridge Univ. Press, Cambridge, 2000.

[17] XI, L. F., and Y. XIONG: Lipschitz equivalence of fractals generated by nested cubes. - Math. Z. 271, 2012, 1287-1308.

Received 27 August 2012 • Accepted 5 October 2012 